\title{
Dietary Supplements and Sports Performance: Minerals
}

\author{
Melvin H. Williams, Ph.D., FACSM \\ Department of Exercise Science, Old Dominion University. Address correspondence to \\ profrunner@aol.com
}

Received April 20, 2005 /Accepted May 15, 2005/Published (online)

\section{ABSTRACT}

Minerals are essential for a wide variety of metabolic and physiologic processes in the human body. Some of the physiologic roles of minerals important to athletes are their involvement in: muscle contraction, normal hearth rhythm, nerve impulse conduction, oxygen transport, oxidative phosphorylation, enzyme activation, immune functions, antioxidant activity, bone health, and acid-base balance of the blood. The two major classes of minerals are the macrominerals and the trace elements. The scope of this article will focus on the ergogenic theory and the efficacy of such mineral supplementation. Journal of the International Society of Sports Nutrition. 2(1):4349, 2005.

Key Words: sport nutrition, dietary supplements, minerals, sports performance

\section{INTRODUCTION}

This is the second in a series of six articles to discuss the major classes of dietary supplements (vitamins; minerals; amino acids; herbs or botanicals; metabolites, constituents/extracts, or combinations). The major focus is on efficacy of such dietary supplements to enhance exercise or sport performance.

\section{MINERALS: ERGOGENIC THEORY}

Minerals represent a class of inorganic substances found naturally in a variety of foods. The human body needs about twenty different minerals in order to function properly. The two major classes of minerals are the macrominerals and the trace elements. In the United States, Recommended Dietary Allowances (RDAs) and Adequate Intakes (AIs) have been developed for 3 macrominerals and 9 trace elements.
Minerals are essential for a wide variety of metabolic and physiologic processes in the human body. Speich and others recently reviewed the physiological roles of minerals important to athletes, noting that minerals are involved in muscle contraction, normal hearth rhythm, nerve impulse conduction, oxygen transport, oxidative phosphorylation, enzyme activation, immune functions, antioxidant activity, bone health, and acidbase balance of the blood ${ }^{1}$. Because many of these processes are accelerated during exercise, an adequate amount of minerals is necessary for optimal functioning. Athletes should obtain an adequate amount of all minerals in their diet, for a mineral deficiency may impair optimal health, and health impairment may adversely affect sport performance. Maughan and others note that iron and calcium are the two micronutrients most likely to be low in the diet, particularly in young athletes ${ }^{2}$. In support of this viewpoint, Ziegler and others recently noted that dietary intake of both iron and calcium was inadequate in female 
figure skaters during the competitive season 3. Although all minerals may play a role in a variety of metabolic and physiologic processes, this presentation will focus on those minerals that have received research attention or consideration relative to effects on physical performance or health of the athlete.

\section{MINERAL SUPPLEMENTS: EFFICACY}

Calcium. About 99 percent of the calcium in the body is stored in the skeletal system, while the remaining one percent is present in other cells, such as muscle cells. Although this muscle cell calcium is involved in a variety of physiologic processes associated with energy metabolism and muscle contraction, calcium supplementation is not considered to possess ergogenic potential because, if necessary, the muscle cells may draw on the vast reserves stored in the bone tissue. However, as noted above, young women involved in weight-control sports, such as figure skating and distance running, may have inadequate dietary intake of calcium. Additionally, exercise may increase calcium losses. For example, Dressendorfer ${ }^{4}$ and others examined the effects of 10-week intense endurance training, including volume, interval and tapering phases, on serum and urinary minerals levels. They found that urinary calcium increased and serum calcium decreased below the clinical norm following the high-intensity interval phase, but these changes were reversed following the tapering phase. Thus, it appears that calcium excretion may be increased with high-intensity training.

Inadequate calcium intake and increased calcium losses may predispose one to osteoporosis. This may be especially so in women who develop the female athlete triad (disordered eating, amenorrhea, osteoporosis). For example, Gremion and others ${ }^{5}$ recently noted that long-distance runners with oligo-amenorrhea had greater decreases in bone mineral density in the spine than in the femur, even though they had similar energy, calcium and protein intakes compared to eumenorrheic runners. The National Institutes of Health consensus panel on osteoporosis indicated that supplementation with calcium, along with vitamin $\mathrm{D}$, may be necessary in persons not achieving the recommended dietary intake such as these female athletes ${ }^{6}$. Additionally, athletes with amenorrhea should consult with their physician regarding the need for drug or hormonal therapy to help prevent osteoporosis.

Phosphates. Phosphates are incorporated into many compounds in the body that are involved in energy metabolism, such as ATP as an energy substrate, thiamin pyrophosphate as a vitamin cofactor, sodium phosphate as a buffer, and 2,3diphosphoglycerate (2,3-DPG) for RBC function. All of these roles could provide ergogenic potential, but the most researched theory involves the effect of phosphate salt supplementation on 2,3-DPG. Increased levels of 2,3-DPG could facilitate release of oxygen from hemoglobin in the red blood cell and possibly enhance aerobic endurance exercise performance. Previous studies have generally, but not universally, shown that phosphate salt supplementation increases serum 2-3-DPG levels. Most recently, Bremner and others found that a 7-day phosphate loading protocol would increase erythrocyte phosphate pools and 2,3-DPG ${ }^{7}$.

About a dozen studies have been conducted with phosphate salt supplementation and their effects on physical performance, and the results are clearly equivocal. However, no study has reported decreases in performance, and four studies from independent laboratories have shown remarkable similarities relative to increased levels of $\mathrm{VO}_{2}$ max following phosphate supplementation and improved performance on bicycle ergometer exercise tests such as a simulated 40-kilometer cycle time trial ${ }^{8-11}$. Although these results are impressive, 
Tremblay and others ${ }^{12}$ indicated that a number of confounding variables in previous research have been identified and more controlled research has been recommended. Research conducted during the past 10 years has also been equivocal, but some favorable effects have been shown. For example, Goss and others recently reported that although phosphate supplementation did not affect physiological responses during exercise at about 70-80 percent VO2max, the rating of perceived exertion (RPE) was lower suggesting a beneficial psychological effect ${ }^{13}$.

Magnesium. Magnesium is a component of over 300 enzymes, some involved in the regulation of muscle contraction, oxygen delivery, and protein synthesis. Several studies have investigated the effect of magnesium supplementation on performance. Lukaski noted that some earlier studies have shown that magnesium supplementation improved strength and cardiorespiratory function in healthy persons and athletes, but also noted it is unclear as to whether these observations related to improvement of an impaired nutritional status or a pharmacologic effect ${ }^{14,15}$. Lukaski also noted that the study designs limit conclusions about dietary recommendations needed to optimize performance. Newhouse and Finstad ${ }^{16}$ also noted that interpretation of the available studies was confounded by differences in research design. Nevertheless, in their metaanalysis of human supplementation studies, they concluded that the strength of the evidence favors those studies finding no effect of magnesium supplementation on any form of exercise performance, including aerobic, anaerobic-lactic acid, and strength activities.

Iron. Iron is one of the most critical minerals with implications for sports performance. Iron is a component of hemoglobin, myoglobin, cytochromes, and various enzymes in the muscle cells, all of which are involved in the transport and metabolism of oxygen for aerobic energy production during endurance exercise. The benefits of iron supplementation may depend on the iron status of the athlete.

Iron Deficiency Anemia. In their review, Beard and Tobin indicated that the prevalence of iron deficiency anemia is likely to be higher in athletic groups, especially in younger female athletes, than in healthy sedentary individuals ${ }^{17}$. They note that it is likely that dietary choices explain much of a negative iron balance, but there may be other reasons as well. For example, in a recent study Jones and others reported hematuria in middle-distance track athletes after intense interval workouts ${ }^{18}$. Hematuria was observed after 45 percent of the workouts, and 90 percent of the athletes experienced post-workout hematuria at least once. Moreover, Schumacher and others compared blood markers of iron status among various male athletic groups and found a marker in endurance runners, as compared to endurance cyclists, that suggested runners may experience more hemolysis due to foot impact ${ }^{19}$. Other possible causes of iron loss in athletic individuals include myoglobin leakage, gastrointestinal losses, sweat losses, and heavy menstrual losses ${ }^{20}$. Such losses could eventually lead to iron deficiency anemia.

Iron deficiency anemia impairs muscular performance. In their review of 29 research reports, Haas and Brownlie ${ }^{21}$ noted a strong causal effect of iron deficiency anemia to impair work capacity in both humans and animals. If an athlete fatigues easily or does not perform up to expectations, iron deficiency anemia may be suspected. A blood analysis should be undertaken for determination of hemoglobin status. If irondeficiency anemia is detected, a physician would prescribe iron supplementation to help restore normal hemoglobin levels. Correcting an iron-deficiency anemia should improve performance. 
Iron deficiency without anemia. Irondeficiency without anemia is a condition of normal hemoglobin levels, but reduced levels of serum ferritin. Serum ferritin levels in the range of 20-30 nanograms per milliliter have been considered markers of iron deficiency. Iron supplementation will usually restore serum ferritin to normal, but studies have indicated that iron supplementation may or may not have an effect on performance.

In their review, Garza and others noted that although iron supplementation can raise serum ferritin, increases in ferritin unaccompanied by increases in hemoglobin concentration have not been shown to improve endurance performance ${ }^{22}$. Although Nielsen and Nachtigall agree with this finding, they note that many of the studies did not meet the general recommendations for the optimal clinical management of iron deficiency ${ }^{23}$. Moreover, several studies from Cornell University have shown that untrained, irondepleted, nonanemic women who received an iron supplement during exercise training had significantly greater increases in $\mathrm{VO}_{2} \mathrm{max}$ and 15-kilometer cycling endurance performance as compared to the placebo group, suggesting that iron deficiency without anemia impairs favorable adaptation to aerobic exercise ${ }^{24,25}$.

Iron saturated. Iron supplements will not enhance performance in athletes with normal hemoglobin and iron status. However, endurance athletes with normal hemoglobin status who attempt to increase their red blood cells (RBC) and hemoglobin levels may benefit from iron supplementation. World class athletes, such as Tour de France champion Lance Armstrong, have been reported to use the technique of "Live high, train low" to stimulate RBC production. Athletes remain at sea level to train intensely, but live in specially constructed altitude houses to stimulate production of natural erythropoietin (EPO) that promotes RBC genesis ${ }^{26}$. Such athletes may benefit from iron supplementation.

Zinc. Zinc is a component of over 300 enzymes, some involved in functions important to physical performance, such as muscle energy production and protein synthesis. Micheletti notes that endurance athletes who adopt a diet rich in carbohydrate but low in protein and fat may decrease zinc intake, which over time may lead to a zinc deficiency with loss of body weight, latent fatigue, and decreased endurance $^{27}$. Although some studies have reported hypozincemia in athletes, particularly endurance athletes, no deficiency symptoms have been noted. Additionally, no data are available to indicate zinc supplementation to such athletes will enhance physical performance 20. Research involving zinc supplementation and exercise performance is very limited, and Lukaski ${ }^{14,15}$ noted that study designs limit our ability to provide recommendations regarding zinc supplementation to athletes.

Chromium. Chromium is an insulin cofactor, and its theorized ergogenic effect is based on the role of insulin to facilitate BCAA transport into the muscle. Chromium has been advertised for strength-type athletes, but also could be theorized to enhance aerobic endurance performance.

Some early research data do suggest an increase in lean body mass and decreased body fat with chromium picolinate supplementation ${ }^{28}$. However, this report was based on unpublished, flawed studies that have not been subjected to the peerreview process. More contemporary research with better experimental design replicated these studies and has shown that chromium picolinate supplementation does not increase lean muscle mass or decrease body fat ${ }^{29-32}$. Other research also indicated different forms of chromium, such as chromium chloride, had no effect on body composition ${ }^{33}$. Chromium supplementation 
has also shown no beneficial effects on sports performance. Walker and others ${ }^{32}$, in an excellent study with highly trained wrestlers, reported no ergogenic effects of chromium picolinate on neuromuscular or metabolic performance, while Livolsi and others ${ }^{34}$, using well-trained female softball players, reported no significant ergogenic effect of chromium picolinate supplementation on muscular strength. Davis and others evaluated the effects of adding chromium to a carbohydrateelectrolyte sports drink on prolonged intermittent high-intensity exercise to fatigue, and found that the chromium picolinate provided no additional advantage above that provided by the carbohydrate ${ }^{35}$. Thus, chromium supplementation does not appear to enhance body composition or performance in well-trained individuals.

Boron. Boron has been marketed as an anabolic mineral, theoretically by increasing serum testosterone. However, this theory was based on research with elderly women and apparently has no application to healthy athletes. Research is limited, but does not document an anabolic effect of boron supplementation $^{36}$.

Vanadium. Vanadium has also been advertised for its anabolic potential, purportedly by enhancement of insulin activity. Some research has shown beneficial effects of vanadium supplementation to type 2 diabetics. However, Jentjens and Jeukendrup recently reported no significant effect of vanadyl sulfate supplementation on insulin sensitivity in healthy adults ${ }^{37}$. Moreover, the limited data available do not support an anabolic effect of vanadium in young healthy individuals ${ }^{38}$.

Selenium. Selenium is a component of several enzymes, particularly glutathione peroxidase (GPx), an important cellular antioxidant enzyme. Theoretically, selenium supplementation could prevent peroxidation of the RBC membrane and muscle cell substructures involved in oxygen metabolism, possibly enhancing aerobic exercise performance. Although antioxidant supplements have not universally been shown to prevent lipid peroxidation, some studies with selenium supplementation have shown an enhancement of GPx status and reduced lipid peroxidation in prolonged aerobic exercise; however, in these studies, actual endurance performance did not improve ${ }^{20}$.

\section{MINERAL SUPPLEMENTS: SAFETY, LEGALITY AND ETHICALITY}

Most mineral supplements are safe in recommended dosages. A Tolerable Upper Intake Level (UL) has been established for 14 minerals. The UL is the maximal level of daily nutrient intake that is likely to pose no risk of adverse effects. With use of fortified foods and mineral dietary supplements, it may be relatively easy for athletes to exceed the UL for several minerals. Most minerals possess significant health risks if consumed in excess. For example, excess dietary iron may lead to hemochromatosis and eventual deterioration of liver function, while excess zinc may decrease HDL-cholesterol levels and increase cardiovascular disease risk. The International Olympic Committee (IOC) does not prohibit use of mineral supplements, so their use is both legal and ethical.

\section{MINERAL SUPPLEMENTS AND SPORT/EXERCISE PERFORMANCE: SUMMARY}

A mineral deficiency may impair performance. In particular, correcting an iron-deficiency anemia will improve aerobic endurance performance. As female athletes are more prone to iron deficiency, the USOC recommends female athletes undergo blood testing periodically to determine hemoglobin status. Optimal calcium nutrition for bone health is also important for female athletes, particularly those in weight-control sports. 
In general, supplementation with calcium, magnesium, iron, zinc, copper, and selenium does not enhance sport performance in wellnourished athletes. Chromium, boron and vanadium have been studied as potential anabolics by potentiating the effects of insulin or testosterone, but studies have reported no beneficial effects of supplementation on body composition or muscular strength and endurance. Significant improvements in maximal oxygen uptake and/or aerobic endurance performance following phosphate salt supplementation have been reported in four well-controlled studies, but more controlled research has been recommended.

\section{REFERENCES}

1. Speich, M., et al. Minerals, trace elements and related biological variables in athletes and during physical activity. Clinical Chimica Acta, v. 312, p. 1-11, 2001.

2. Maughan, R., et al. Nutrition and the young athlete. Medicina Sportiva, v.4, p.E51-58, 2000.

3. Ziegler, P., et al. Nutritional status of teenage female competitive figure skaters. Journal of the American Dietetic Association, v. 102, p. 374-79, 2002.

4. Dressendorfer, R., et al. Mineral metabolism in male cyclists during high-intensity endurance training. International Journal of Sport Nutrition and Exercise Metabolism,v.12, p.63-72, 2002.

5. Gremion, G., et al. Oligo-amenorrheic long-distance runners may lose more bone in spine than in femur. Medicine \& Science in Sports \& Exercise. v. 33, p. 15-21, 2001.

6. NIH Consensus Development Panel on Osteoporosis Prevention, Diagnosis, and Therapy. Osteoporosis prevention, diagnosis, and therapy. JAMA, v. 285, p. 785-95, 2001.

7. Bremner, K., et al. The effect of phosphate loading on erythrocyte 2,3-bisphophoglycerate levels. Clinical Chimica Acta, v. 323, p.111-14, 2002.

8. Cade, R., et al. Effects of phosphate loading on 2, 3-diphosphoglycerate and maximal oxygen uptake. Medicine \& Science in Sports \& Exercise, v. 16, p. 263-68, 1984

9. Kreider, R., et al. Effects of phosphate loading on oxygen uptake, ventilatory anaerobic threshold, and run performance. Medicine \& Science in Sports \& Exercise, v. 22, p. 250-56, 1990

10. Kreider, R., et al. Effects of phosphate loading on metabolic and myocardial responses to maximal and endurance exercise. International Journal of Sport Nutrition, v. 2, p. 20-27, 1992

11. Stewart, I., et al. Phosphate loading and the effects on $\mathrm{VO}_{2} \max$ in trained cyclists. Research Quarterly for Exercise and Sport, v. 61, p. 80-84, 1990

12. Tremblay, M., et al. Ergogenic effects of phosphate loading: Physiological fact or methodological fiction? Canadian Journal of Applied Physiology, v. 19, p.1-11, 1994.

13. Goss, F., et al. Effect of potassium phosphate supplementation on perceptual and physiological responses to maximal graded exercise. International Journal of Sport Nutrition and Exercise Metabolism, v. 11, p. 53-62, 2001.

14. Lukaski, H. Magnesium, zinc, and chromium nutrition and athletic performance. Canadian Journal of Applied Physiology, v. 26, p. S13-22, 2001

15. Lukaski, H. Magnesium, zinc, and chromium nutriture and physical activity. American Journal of Clinical Nutrition, v. 72, p 585S-93S, 2000.

16. Newhouse, I., and Finstad, E. The effects of magnesium supplementation on exercise performance. Clinical Journal of Sport Medicine, v. 10, p. 195-200, 2000.

17. Beard, J., and Tobin, B. Iron status and exercise. American Journal of Clinical Nutrition, v. 72, p. 594S-97S, 2000.

18. Jones, G., et al. The incidence of hematuria in middle distance track running. Canadian Journal of Applied Physiology, v. 26, p. 336-49, 2001.

19. Schumacher, Y., et al. Hematological indices and iron status in athletes of various sports and performances. Medicine \& Science in Sports \& Exercise, v. 34, p. 869-75, 2002.

20. Williams, M. H. Nutrition for Health, Fitness \& Sport ( $7^{\text {th }}$ edition). New York: McGraw-Hill, 2004.

21. Haas, J., and Brownlie, T. Iron deficiency and reduced work capacity: A critical review of the research to determine a causal relationship. Journal of Nutrition, v. 131, p. 676S-88S, 2001.

22. Garza, D., et al. The clinical value of serum ferritin tests in endurance athletes. Clinics in Sports Medicine, v. 7, p. 46-53, 1997.

23. Nielsen, P., and Nachtigall, D. Iron supplementation to athletes: Current recommendations. Sports Medicine, v. 26, p. 207-16, 1998.

24. Brownlie, T., et al. Marginal iron deficiency without anemia impairs aerobic adaptation among previously untrained women. American Journal of Clinical Nutrition, v. 75, p. 734-42, 2002.

25. Hinton, $\mathrm{P}$., et al. Iron supplementation improves endurance after training in iron-depleted nonanemic women. Journal of Applied Physiology, v. 88, p. 1103-11, 2000.

26. Runner's World. Live high, train low. Runner's World, v. 38, n. 1, p. 64, 2003.

27. Micheletti, A., et al. Zinc status in athletes: Relation to diet and exercise. Sports Medicine, v. 31, p. 577-82, 2001. 
28. Evans, G. W. The effect of chromium picolinate on insulin controlled parameters in humans. International Journal of Biosocial and Medical Research, v. 11, p. 163-180, 1989.

29. Clancy, S. P., et al. Effects of chromium picolinate supplementation on body composition, strength, and urinary chromium loss in football players. International Journal of Sport Nutrition, v. 4, p. 142-153, 1994.

30. Hallmark, M. A., et al. Effects of chromium supplementation and resistive training on muscle strength and lean body mass in untrained men. Medicine \& Science in Sports \& Exercise, v. 28, p. 139-44, 1993.

31. Trent, L., and Thieding-Cancel, D. Effects of chromium picolinate on body composition. Journal of Sports Medicine and Physical Fitness, v. 35, p. 273-80, 1995.

32. Walker, L., et al. Chromium picolinate effects on body composition and muscular performance in wrestlers. Medicine \& Science in Sports \& Exercise, v. 30, p. 1730-37, 1998.

33. Lukaski, H., et al. Chromium supplementation and resistance training: Effects on body composition, strength, and trace element status of men. American Journal of Clinical Nutrition, v. 63, 954-65, 1996

34. Livolsi, J., et al. The effect of chromium picolinate on muscular strength and body composition in women athletes. Journal of Strength and Conditioning Research, v. 15, p. 161-6, 2001.

35. Davis, J., et al. Effects of carbohydrate and chromium ingestion during intermittent high-intensity exercise to fatigue. International Journal of Sport Nutrition and Exercise Metabolism, v. 10, p. 476-85, 2000.

36. Ferrando, A., and Green, N. The effect of boron supplementation on lean body mass, plasma testosterone levels, and strength in male bodybuilders. International Journal of Sport Nutrition, v. 3, p. 140-49, 1993.

37. Jentjens, R., and Jeukendrup, A. Effect of acute and short-term administration of vanadyl sulphate on insulin sensitivity in healthy active humans. International Journal of Sport Nutrition and Exercise Metabolism, v. 12, p. 470-79, 2002.

38. Fawcett, J., et al. The effect of oral vanadyl sulfate on body composition and performance in weight-training athletes. International Journal of Sport Nutrition, v. 6, p. 382-90, 1996 